This document is published in:

Composite Structures, (2013), 104, 154-161.

DOI: http://dx.doi.org/10.1016/j.compstruct.2013.04.022

(C) 2013 Elsevier Ltd. 


\title{
Axisymmetric free vibration of closed thin spherical nano-shell
}

\author{
R. Zaera, J. Fernández-Sáez * J.A. Loya \\ Department of Continuum Mechanics and Structural Analysis, University Carlos III of Madrid, Avda. de la Universidad, 30, 28911 Leganés, Madrid, Spain \\ * Corresponding author. Tel.: +34 916249964; fax: +34 916249430. \\ E-mail address: ppfer@ing.uc3m.es (J. Fernández-Sáez).
}

\begin{abstract}
This work investigates the free axisymmetric vibrations of a closed spherical nano-shell using the Eringen nonlocal elasticity theory. The motion equations are properly formulated considering the hypotheses of thin shells and the solution is obtained using the classical separation of variables method. The effect of the nonlocal parameter on the natural frequencies and modal shapes are discussed in comparison to their local counterparts. This study could be useful in biomedical and bioengineering applications as well as in other fields related with the nanotechnology.
\end{abstract}

Keywords: Nonlocal elasticity, Sphere vibration, Nanotechnology, Natural frequencies, Modal shapes

\section{Introduction}

Modern technological applications involve the use of systems which can be devised as micro or nanostructures. From the discovery of fullerenes [1] and Carbon Nanotubes (CNTs) [2], these applications have experienced an exponential growth, mainly in micro-or nano-electromechanical (MEMS or NEMS) devices [3], nanomachines [4-7], as well as in biotechnology and biomedical fields [8].

A main characteristic of these nanostructures is that their dimensions become comparable to the size of their material microstructure or the molecular distances, thus the size effects are significant regarding their mechanical behavior. These systems could be analysed using Molecular Dynamics [9-12]. Since the atomic and molecular approaches require a great computational effort, simplified models are useful for analysing the mechanical behavior of such devices. However, classical continuum mechanics cannot predict the size effect, because the constitutive equations derived from this framework lack an internal length, characterizing the underlying microstructure, i.e. it is a scale-free theory.

Despite some sporadic efforts in the 19th century due to Cauchy and Voigt, and in the first half of the 20th century through the work of the Cosserat brothers to capture the effects of microstructure using the continuum equations of elasticity with additional higher-order derivatives, the major revival took place in the 1960s. From this time are the works of Mindlin and Tiersten [13], Kröner [14], Toupin [15,16], Green and Rivlin [17], Mindlin $[18,19]$ and Mindlin and Eshel [20]. However, these formulations were excessively complex with too many parameters and equations. For instance, the more general form of the constitutive tensors proposed by Mindlin [18] includes 903 independent elastic constants that can be reduced to 18 for the isotropic case.
More recently, Eringen derived, from his earlier integral nonlocal theories [21], a simple stress-gradient formulation which contains a length scale parameter. In the early 1990s, Aifantis and coworkers suggested to extend the linear elastic constitutive relations with the Laplacian of the strain through a length scale parameter again [22-24]. Askes and Gitman [25] shown that an unification of both Eringen and Aifantis theories is possible. An overview on the historical development of theses theories, as well as its meaning and implementation can be found in the paper by Askes and Aifantis [26].

Among the size-dependent continuum theories, the theory of nonlocal continuum mechanics initiated by Eringen and coworkers $[27,28,21]$ has been widely used to analyse many problems, such as wave propagation, dislocation, and crack singularities. From the pioneer work of Peddieson et al. [29], this theory has been also used to solve problems involving nanostructures. Thus, the Eringen nonlocal theory of elasticity has been used to address the behavior of beams [30-36], beams under rotation [37-40], rods [41-46], plates [47-49], cylindrical shells [50-52], conical shells $[53,11,12]$, rings [54,55] and particles [56], as well as carbon nanotubes (CNTS) [57-64].

On the other hand, the dynamics of the closed spherical shells (fluid-filled or empty) is a problem of technological importance in some modern industrial, biomedical, biological and other applications $[65,66]$. There exists an extensive bibliography dedicated to the analysis of buckling and vibrations of spherical shells from the point of view of the classical elasticity theory [67-70]. The classical continuum framework has been also applied to the case of nanospheres [71,72]. In various modern biomedical and biological applications, spherical membrane structure (fluid-filled or empty) can be used to model some micro/nanosized components, which 
are used as targeted drug delivery systems [73], biological cells [74], and some kind of viruses [75,76].

The above cited works rest on the concepts of classical continuum mechanics. In the authors knowledge, the only analyses concerning spherical shells which take into account size effects using nonlocal continuum theories is due to Ghavanloo and Fazelzadeh [77], who presented a study on the radial vibrations of a closed spherical shell using the Eringen nonlocal elasticity theory, and the same authors [66], who studied the coupled axisymmetric vibrations of fluid-filled closed spherical membrane shell using the same nonlocal approach. However, for the case of axisymmetric vibrations, its formulation of the problem is not properly addressed because some issues concerning the handling of several differential operators in spherical coordinates are omitted and the analysis should be revisited.

In this paper we present a detailed study of free axisymmetric vibrations of a closed spherical nano-shell using the Eringen nonlocal elasticity theory. The hypothesis of thin shells have been taken into account and then bending moments, shear efforts and radial normal stresses were neglected. The solution method proposed follows the procedure used by Baker [68] for the elastic local case. The effect of the nonlocal parameter on the results, i.e. natural frequencies and mode shapes, are discussed.

\section{General equations of Eringen elasticity theory}

The Eringen formulation $[28,78,79]$ states that the nonlocal stress-tensor $\mathbf{t}$ at any point $\mathbf{x}$ in a body can be expressed as

$\mathbf{t}(\mathbf{x})=\int_{\Omega} \alpha\left(\left|\mathbf{x}^{\prime}-\mathbf{x}\right|, \gamma\right) \boldsymbol{\sigma}(\mathbf{x}) d \Omega\left(\mathbf{x}^{\prime}\right)$

where $\boldsymbol{\sigma}(\mathbf{x})$ is the classical local stress tensor at point $\mathbf{x}$, which is related to the linear strain tensor $\boldsymbol{\varepsilon}$ by the conventional constitutive relations for a Hookean material

$\boldsymbol{\sigma}=\mathbf{C}: \boldsymbol{\varepsilon}$

where $\mathbf{C}$ is the fourth-order elasticity tensor and $\boldsymbol{\varepsilon}$ is given by

$\boldsymbol{\varepsilon}=\frac{1}{2}\left(\nabla \mathbf{u}+\nabla\left(\mathbf{u}^{T}\right)\right)$

$\mathbf{u}$ being the displacement vector.

Eq. (1) represents the weighted average of the contributions to the stress field at a point $\mathbf{x}$ of the strain field of all points in the body in the neighborhood of $\mathbf{x}$, the size of which is related to the nonlocal modulus $\alpha\left(\left|\mathbf{x}^{\prime}-\mathbf{x}\right|, \gamma\right)$. Here, $\left|\mathbf{x}^{\prime}-\mathbf{x}\right|$ is the Euclidean distance and $\gamma$ is a material constant given by $\gamma=e_{0} a / l$, that depends on internal and external characteristic lengths ( $a$ and $l$, respectively) trough an adjusting constant $e_{0}$, dependent on each material.

Both Eqs. (1) and (2) define the considered nonlocal constitutive behavior of a Hookean solid. For a class of physically admissible kernel $\alpha\left(\left|\mathbf{x}^{\prime}-\mathbf{x}\right|, \gamma\right)$, Eringen [21] showed that the nonlocal constitutive equations given by the integral formulation could be replaced by gradients. Thus, Eq. (1) can be written in an equivalent differential form as

$\left(1-\kappa^{2} \nabla^{2}\right) \mathbf{t}=\boldsymbol{\sigma}$

$\kappa=e_{0} a$ being the length scale which takes into account the size effect on the response of nanostructures.

The balance of linear momentum results in the following equation of motion

$\nabla \cdot \mathbf{t}+\mathbf{f}=\rho \ddot{\mathbf{u}}$

where $\mathbf{f}$ represents the external body forces vector, and after using Eq. (4)

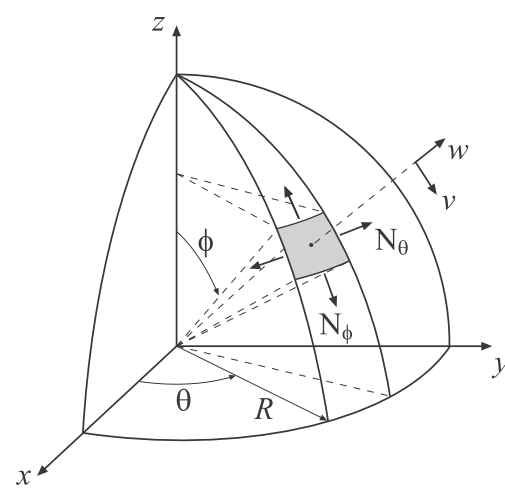

Fig. 1. Differential element of shell, membrane forces and symmetric displacements in spherical coordinates.

$\nabla \cdot \boldsymbol{\sigma}+\mathbf{f}=\left(1-\kappa^{2} \nabla^{2}\right) \rho \ddot{\mathbf{u}}$

Note that the displacement field of a nonlocal solid subject to an external body force field $\mathbf{f}$ and an inertial body force $-\rho \ddot{\mathbf{u}}$ is the same as that of a classical solid subject to the same external force f and an inertial body force $-\left(1-\kappa^{2} \nabla^{2}\right) \rho \ddot{\mathbf{u}}$.

Considering that the material is isotropic, the equations of motion can be obtained in terms of the displacements

$\left(1-\kappa^{2} \nabla^{2}\right) \rho \ddot{\mathbf{u}}=\left(\lambda_{L}+G\right) \nabla(\nabla \cdot \mathbf{u})+G \nabla^{2} \mathbf{u}+\mathbf{f}$

$\lambda_{L}$ and $G$ being the Lamé constants. The above relation constitutes the Navier equation of motion for nonlocal solids, which must be solved with the appropriate initial and boundary conditions applicable in each case.

\section{Axisymmetric motion for the nonlocal spherical shell}

The above theory will be used to study the axisymmetric free vibration of a nonlocal closed thin spherical shell.

\subsection{Problem formulation}

We assume that the deformations are small enough for linear equations to adequately describe the motion. The thickness $h$ of the shell is thin enough that bending moments, shear forces and radial normal stresses can be neglected. The sign convention for radial $w(\phi, t)$ and meridional $v(\phi, t)$ displacements, and for stress resultants in meridional $N_{\phi}(\phi, t)$ and circumferential $N_{\theta}(\phi, t)$ directions, are defined in Fig. 1. The governing equations of motion for the nonlocal spherical shell can be directly derived from the classical formulation for local elasticity [68], as a result of the aforementioned analogy between the nonlocal solid subjected to an inertial body force field $-\rho \ddot{\mathbf{u}}$ and the equivalent local solid subjected to an inertial body force field $-\left(1-\kappa^{2} \nabla^{2}\right) \rho \ddot{\mathbf{u}}$. Neglecting the external body force $\mathbf{f}$, in order to consider free vibrations, the corresponding equations may be expressed as

$$
\begin{aligned}
& -\left(\widehat{N}_{\phi}+\widehat{N}_{\theta}\right) \\
& \left.\left.=\rho R h \frac{\partial^{2} w}{\partial t^{2}}-\frac{\kappa^{2}}{R^{2}} \frac{\partial^{4} w}{\partial t^{2} \partial \phi^{2}}+\cot \phi \frac{\partial^{3} w}{\partial t^{2} \partial \phi}-2 \frac{\partial^{2} w}{\partial t^{2}}-2 \frac{\partial^{3} v}{\partial t^{2} \partial \phi}-2 \cot \phi \frac{\partial^{2} v}{\partial t^{2}}\right)\right)
\end{aligned}
$$

$\frac{\partial \widehat{N}_{\phi}}{\partial \phi}+\cot \phi\left(\widehat{N}_{\phi}-\widehat{N}_{\theta}\right)$

$\left.\left.=\rho R h \frac{\partial^{2} v}{\partial t^{2}}-\frac{\kappa^{2}}{R^{2}} \frac{\partial^{4} v}{\partial t^{2} \partial \phi^{2}}+\cot \phi \frac{\partial^{3} v}{\partial t^{2} \partial \phi}-\frac{1}{\sin ^{2} \phi} \frac{\partial^{2} v}{\partial t^{2}}+2 \frac{\partial^{3} w}{\partial t^{2} \partial \phi}\right)\right)$ 
where the proper differential operators in spherical coordinates have been used. $R, \rho, E$ and $v$ are the sphere radius, mass density, Young modulus and Poisson ratio respectively. $\widehat{N}_{\phi}$ and $\widehat{N}_{\theta}$ are the stress resultants in the equivalent local problem, that can be expressed in terms of the displacements as can be found, for instance, in Soedel [70]

$\widehat{N}_{\phi}=\frac{E h}{R\left(1-v^{2}\right)}\left(\frac{\partial v}{\partial \phi}+w+v(\cot \phi v+w)\right)$

$\widehat{N}_{\theta}=\frac{E h}{R\left(1-v^{2}\right)}\left(\cot \phi v+w+v\left(\frac{\partial v}{\partial \phi}+w\right)\right)$

Considering the following nondimensional quantities

$\bar{v}=\frac{v}{R} ; \quad \bar{w}=\frac{w}{R} ; \quad \tau=\frac{c_{s} t}{R} ; \quad c_{s}=\sqrt{\frac{E}{\rho\left(1-v^{2}\right)}} ; \quad \mu=\frac{\kappa}{R}$

the nondimensional equations of motion may be finally written in terms of displacements as

$$
\begin{aligned}
& -(1+v)\left(\frac{\partial \bar{v}}{\partial \phi}+\cot \phi \bar{v}+2 \bar{w}\right) \\
& \left.=\frac{\partial^{2} \bar{w}}{\partial \tau^{2}}-\mu^{2} \frac{\partial^{4} \bar{w}}{\partial \tau^{2} \phi^{2}}+\cot \phi \frac{\partial^{3} \bar{w}}{\partial \tau^{2} \phi}-2 \frac{\partial^{2} \bar{w}}{\partial \tau^{2}}-2 \frac{\partial^{3} \bar{v}}{\partial \tau^{2} \partial \phi}-2 \cot \phi \frac{\partial^{2} \bar{v}}{\partial \tau^{2}}\right) \\
& \frac{\partial^{2} \bar{v}}{\partial \phi^{2}}+\frac{\partial \bar{v}}{\partial \phi} \cot \phi-\left(v+\cot ^{2} \phi\right) \bar{v}+(1+v) \frac{\partial \bar{w}}{\partial \phi} \\
& \left.=\frac{\partial^{2} \bar{v}}{\partial \tau^{2}}-\mu^{2} \frac{\partial^{4} \bar{v}}{\partial \tau^{2} \phi^{2}}+\cot \phi \frac{\partial^{3} \bar{v}}{\partial \tau^{2} \phi}-\csc ^{2} \phi \frac{\partial^{2} \bar{v}}{\partial \tau^{2}}+2 \frac{\partial^{3} \bar{w}}{\partial \tau^{2} \phi}\right)
\end{aligned}
$$

These equations are identical to the local formulation if the nonlocal parameter $\mu$ is set to zero. Note that, for $\mu>0$, Eqs. (13) and (14) are not coincident with those proposed by Fazelzadeh and Ghavanloo [66].

Following Baker [68] we will assume that the shell is initially at rest in a deformed shape, according to the following initial conditions

$\begin{array}{ll}\bar{w}(\phi, 0)=f(\phi) ; & \left.\frac{\partial \bar{w}}{\partial \tau}\right|_{\tau=0}=0 \\ \bar{v}(\phi, 0)=g(\phi) ; & \left.\frac{\partial \bar{v}}{\partial \tau}\right|_{\tau=0}=0\end{array}$

Eqs. (13) and (14), subject to the initial conditions (15) and (16), determine the free vibrational movement of the nonlocal spherical shell.

\subsection{Solving method}

The differential equations to be solved are linear and homogeneous. Thus, in order to obtain the natural frequencies and modal shapes of the vibration of the shell, the method of separation of variables will be used. Let us assume

$$
\begin{aligned}
& \bar{w}(\phi, \tau)=\sum_{n} W_{n}(\phi) S_{n}(\tau) \\
& \bar{v}(\phi, \tau)=\sum_{n} V_{n}(\phi) Q_{n}(\tau)
\end{aligned}
$$

Then, Eqs. (13) and (14) become

$$
\begin{aligned}
& -2(1+v) W_{n} S_{n}-(1+v)\left(V_{n}^{\prime}+\cot \phi V_{n}\right) Q_{n} \\
& =\left(W_{n}-\mu^{2}\left(W_{n}^{\prime \prime}+\cot \phi W_{n^{\prime}}-2 W_{n}\right)\right) \frac{d^{2} S_{n}}{d \tau^{2}} \\
& \quad+2 \mu^{2}\left(V_{n}^{\prime}+\cot \phi V_{n}\right) \frac{d^{2} Q_{n}}{d \tau^{2}}
\end{aligned}
$$

$$
\begin{aligned}
(1 & +v) W_{n}^{\prime} S_{n}+\left(V_{n}^{\prime \prime}+\cot \phi V_{n}^{\prime}-\left(v+\cot ^{2} \phi\right) V_{n}\right) Q_{n} \\
& =-2 \mu^{2} W_{n}^{\prime} \frac{d^{2} S_{n}}{d \tau^{2}}+\left(V_{n}-\mu^{2}\left(V_{n}^{\prime \prime}+\cot \phi V_{n}^{\prime}-\csc ^{2} \phi V_{n}\right)\right) \frac{d^{2} Q_{n}}{d \tau^{2}}
\end{aligned}
$$

or, dividing Eq. (19) by $\left(W_{n}-\mu^{2}\left(W_{n}^{\prime \prime}+\cot \phi W_{n}^{\prime}-2 W_{n}\right)\right)$ and Eq. (20) by $\left(V_{n}-\mu^{2}\left(V_{n}^{\prime \prime}+\cot \phi V_{n}^{\prime}-\csc ^{2} \phi V_{n}\right)\right)$

$$
\begin{aligned}
& -\lambda_{1} S_{n}-(1+v) \lambda_{2} Q_{n}=\frac{d^{2} S_{n}}{d \tau^{2}}+2 \mu^{2} \lambda_{2} \frac{d^{2} Q_{n}}{d \tau^{2}} \\
& (1+v) \lambda_{3} S_{n}+\lambda_{4} Q_{n}=-2 \mu^{2} \lambda_{3} \frac{d^{2} S_{n}}{d \tau^{2}}+\frac{d^{2} Q_{n}}{d \tau^{2}}
\end{aligned}
$$

with

$\lambda_{1}=\frac{2(1+v) W_{n}}{W_{n}-\mu^{2}\left(W_{n}^{\prime \prime}+\cot \phi W_{n}^{\prime}-2 W_{n}\right)}$

$\lambda_{2}=\frac{V_{n}^{\prime}+\cot \phi V_{n}}{W_{n}-\mu^{2}\left(W_{n}^{\prime \prime}+\cot \phi W_{n}^{\prime}-2 W_{n}\right)}$

$\lambda_{3}=\frac{W_{n}^{\prime}}{V-\mu^{2}\left(V_{n}^{\prime \prime}+\cot \phi V_{n}^{\prime}-\csc ^{2} \phi V_{n}\right)}$

$\lambda_{4}=\frac{V_{n}^{\prime \prime}+\cot \phi V_{n}^{\prime}-\left(v+\cot ^{2} \phi\right) V_{n}}{V_{n}-\mu^{2}\left(V_{n}^{\prime \prime}+\cot \phi V_{n}^{\prime}-\csc ^{2} \phi V_{n}\right)}$

For Eqs. (21) and (22) to be separable, $\lambda_{i}$ must be constant.

We can now determine the constants $\lambda_{1}$ to $\lambda_{4}$ and the functional forms of $W_{n}$ and $V_{n}$.

\subsection{Determination of $\lambda_{i}$ and functional forms $W_{n}$ and $V_{n}$}

For convenience we let $x=\cos \phi$. Then Eq. (23) leads to the Legendre differential equation [80]

$\left(1-x^{2}\right) \frac{d^{2} W_{n}}{d x^{2}}-2 x \frac{d W_{n}}{d x}+\frac{2\left(1+v-\mu^{2} \lambda_{1}\right)-\lambda_{1}}{\mu^{2} \lambda_{1}} W_{n}=0$

For radial displacements $W_{n}$ to be bounded over the entire sphere, the following condition is required

$\frac{2\left(1+v-\mu^{2} \lambda_{1}\right)-\lambda_{1}}{\mu^{2} \lambda_{1}}=n(n+1)$

or

$\lambda_{1}=\frac{2(1+v)}{1+\mu^{2}(2+n(n+1))}$

and the solution for $W_{n}$ is given by the Legendre polynomials

$W_{n}(x)=P_{n}(x)=\frac{1}{2^{n} n !} \frac{d^{n}\left(x^{2}-1\right)^{n}}{d x^{n}}$

with $n$ integer greater or equal than zero.

In a similar way, Eq. (26) leads to a general Legendre differential equation

$\left(1-x^{2}\right) \frac{d^{2} V_{n}}{d x^{2}}-2 x \frac{d V_{n}}{d x}+\left(\frac{1-v-\lambda_{4}}{1+\mu^{2} \lambda_{4}}-\frac{1}{1-x^{2}}\right) V_{n}=0$

For meridional displacements $V_{n}$ being finite and single-valued over the entire sphere, the following condition is required

$\frac{1-v-\lambda_{4}}{1+\mu^{2} \lambda_{4}}=n(n+1)$

or

$\lambda_{4}=\frac{1-v-n(n+1)}{1+\mu^{2} n(n+1)}$ 
and the solution for $V_{n}$ is given by the associated Legendre Polynomials of the first kind (Condon-Shortley phase ommited, see Arfken et al. [81])

$V_{n}(x)=P_{n}^{1}(x)=\frac{\left(1-x^{2}\right)^{1 / 2}}{2^{n} n !} \frac{d^{n+1}\left(x^{2}-1\right)^{n}}{d x^{n+1}}$

with $n$ integer greater than zero.

To obtain $\lambda_{2}$ we use the following relation between Legendre polynomials and associated Legendre polynomials of the first kind

$P_{n}^{1}(x)=\left(1-x^{2}\right)^{1 / 2} \frac{d P_{n}(x)}{d x}$

which is equivalent to

$V_{n}=-W_{n}^{\prime}$

Substituting Eq. (36) and its derivative into Eq. (24) to eliminate $V_{n}$ and $V_{n}^{\prime}$, we get again the Legendre differential equation

$\left(1-x^{2}\right) \frac{d^{2} W_{n}}{d x^{2}}-2 x \frac{d W_{n}}{d x}-\frac{\lambda_{2}\left(1+2 \mu^{2}\right)}{\mu^{2} \lambda_{2}-1} W_{n}=0$

For $W_{n}$ to be a bounded solution of this equation, the following condition is required

$-\frac{\lambda_{2}\left(1+2 \mu^{2}\right)}{\mu^{2} \lambda_{2}-1}=n(n+1)$

or

$\lambda_{2}=\frac{n(n+1)}{1+\mu^{2}(2+n(n+1))}$

Now substituting Eq. (36) in Eq. (25) to eliminate $W_{n}^{\prime}$, we obtain a general Legendre differential equation

$\left(1-x^{2}\right) \frac{d^{2} V_{n}}{d x^{2}}-2 x \frac{d V_{n}}{d x}+\left(\frac{-1-\lambda_{3}}{\mu^{2} \lambda_{3}}-\frac{1}{1-x^{2}}\right) V_{n}=0$

For $V_{n}$ to be a finite and single-valued solution of this equation, the following condition is required

$\frac{-1-\lambda_{3}}{\mu^{2} \lambda_{3}}=n(n+1)$

or

$\lambda_{3}=-\frac{1}{1+\mu^{2} n(n+1)}$

\subsection{Solution for the time-dependent functions $S_{n}$ and $Q_{n}$}

By suitable combinations of Eqs. (21) and (22), we can transform them in two uncoupled biquadratic differential equations

$\frac{d^{4} S_{n}}{d \tau^{4}}+\xi_{n} \frac{d^{2} S_{n}}{d \tau^{2}}+\gamma_{n} S_{n}=0$

$\frac{d^{4} Q_{n}}{d \tau^{4}}+\xi_{n} \frac{d^{2} Q_{n}}{d \tau^{2}}+\gamma_{n} Q_{n}=0$

Note that both equations have the same coefficients

$\xi_{n}=\frac{(1+n(n+1))\left(1+\mu^{2}(-2+n(n+1))\right)-v\left(-3+\mu^{2}(-2+n(n+1))\right)}{1+\mu^{2}\left[2(1+n(n+1))+\mu^{2} n(n+1)(-2+n(n+1))\right]}$

$\gamma_{n}=\frac{\left(1-v^{2}\right)(-2+n(n+1))}{1+\mu^{2}\left[2(1+n(n+1))+\mu^{2} n(n+1)(-2+n(n+1))\right]}$ as

The solution of these linear differential equations can be written

$$
\begin{aligned}
S_{n}(\tau)= & A_{n}^{S} \cos \left(a_{n} \tau\right)+B_{n}^{S} \sin \left(a_{n} \tau\right)+C_{n}^{S} \cos \left(b_{n} \tau\right)+D_{n}^{S} \sin \left(b_{n} \tau\right) \\
Q_{n}(\tau)= & A_{n}^{Q} \cos \left(a_{n} \tau\right)+B_{n}^{Q} \sin \left(a_{n} \tau\right) \\
& +C_{n}^{Q} \cos \left(b_{n} \tau\right)+D_{n}^{Q} \sin \left(b_{n} \tau\right)
\end{aligned}
$$

where the natural frequencies are roots of the corresponding characteristic equation of the differential problem given by Eqs. (43) and (44)

$a_{n}=\left(\frac{\xi_{n}+\sqrt{\xi_{n}^{2}-4 \gamma_{n}}}{2}\right)^{1 / 2}$

$b_{n}=\left(\frac{\xi_{n}-\sqrt{\xi_{n}^{2}-4 \gamma_{n}}}{2}\right)^{1 / 2}$

As in the local elasticity case, there are two natural frequencies for each value of $n$ : the upper branch $a_{n}$ and the lower branch $b_{n}$. The eight unknown amplitudes in Eqs. (47) and (48) are obtained, applying the corresponding initial conditions, in terms of the initial values of the functions $S_{n}$ and $Q_{n}$ and of their first, second and third derivatives

$S_{n}(0)=S_{n}^{0} ; \quad Q_{n}(0)=Q_{n}^{0}$

$\left.\frac{d S_{n}}{d \tau}\right|_{\tau=0}=\left.\frac{d Q_{n}}{d \tau}\right|_{\tau=0}=0$

$\left.\frac{d^{2} Q_{n}}{d \tau^{2}}\right|_{\tau=0}=\ddot{Q}_{n}^{0}=p_{n} S_{n}^{0}+q_{n} Q_{n}^{0}$

$\left.\frac{d^{2} S_{n}}{d \tau^{2}}\right|_{\tau=0}=\ddot{S}_{n}^{0}=r_{n} S_{n}^{0}+S_{n} Q_{n}^{0}$

and

$\left.\frac{d^{3} S_{n}}{d \tau^{3}}\right|_{\tau=0}=\left.\frac{d^{3} Q_{n}}{d \tau^{3}}\right|_{\tau=0}=0$

with

$p_{n}=\frac{\left(1+v-2 \mu^{2} \lambda_{1}\right) \lambda_{3}}{1+4 \mu^{4} \lambda_{2} \lambda_{3}}$

$q_{n}=\frac{-2 \mu^{2}(1+v) \lambda_{2} \lambda_{3}+\lambda_{4}}{1+4 \mu^{4} \lambda_{2} \lambda_{3}}$

$r_{n}=-\frac{\lambda_{1}+2 \mu^{2}(1+v) \lambda_{2} \lambda_{3}}{1+4 \mu^{4} \lambda_{2} \lambda_{3}}$

$s_{n}=-\frac{\lambda_{2}\left(1+v+2 \mu^{2} \lambda_{4}\right)}{1+4 \mu^{4} \lambda_{2} \lambda_{3}}$

Initial conditions (51) and (52) correspond to Eqs. (15) and (16). Initial conditions (53) and (54) are obtained combining Eqs. (21) and (22), whereas initial conditions (55) are obtained by derivation of Eqs. (21) and (22). The solution of the algebraic system of equations given by the eight initial conditions, Eqs. (51)-(55), leads to

$B_{n}^{S}=D_{n}^{S}=B_{n}^{Q}=D_{n}^{Q}=0$

$A_{n}^{S}=S_{n}^{0} \frac{\ddot{S}_{n}^{0} / S_{n}^{0}+b_{n}^{2}}{b_{n}^{2}-a_{n}^{2}}$ 
$C_{n}^{S}=S_{n}^{0} \frac{\ddot{S}_{n}^{0} / S_{n}^{0}+a_{n}^{2}}{a_{n}^{2}-b_{n}^{2}}$

$A_{n}^{Q}=Q_{n}^{0} \frac{\ddot{Q}_{n}^{0} / Q_{n}^{0}+b_{n}^{2}}{b_{n}^{2}-a_{n}^{2}}$

$C_{n}^{Q}=Q_{n}^{0} \frac{\ddot{Q}_{n}^{0} / Q_{n}^{0}+a_{n}^{2}}{a_{n}^{2}-b_{n}^{2}}$

Taking into account conditions (53) and (54), and the relation $a_{n}^{2}+b_{n}^{2}=\xi_{n}$, the final expression for the time-dependent functions are

$S_{n}(\tau)=S_{n}^{0}\left[\frac{\beta_{n}-a_{n}^{2}}{b_{n}^{2}-a_{n}^{2}} \cos \left(a_{n} \tau\right)+\frac{\beta_{n}-b_{n}^{2}}{a_{n}^{2}-b_{n}^{2}} \cos \left(b_{n} \tau\right)\right]$

$Q_{n}(\tau)=Q_{n}^{0}\left[\frac{\alpha_{n}-a_{n}^{2}}{b_{n}^{2}-a_{n}^{2}} \cos \left(a_{n} \tau\right)+\frac{\alpha_{n}-b_{n}^{2}}{a_{n}^{2}-b_{n}^{2}} \cos \left(b_{n} \tau\right)\right]$

where

$\beta_{n}=\frac{\ddot{S}_{n}^{0}}{S_{n}^{0}}+\xi_{n}$

$\alpha_{n}=\frac{\ddot{Q}_{n}^{0}}{Q_{n}^{0}}+\xi_{n}$

The initial values $S_{n}^{0}$ and $Q_{n}^{0}$ can be obtained following the procedure proposed by Baker [68]. Considering Eqs. (17) and (18) at $t=0$

$\bar{w}(x, 0)=\sum_{n=0}^{\infty} P_{n}(x) S_{n}^{0}$

$\bar{v}(x, 0)=\sum_{n=1}^{\infty} P_{n}^{1}(x) Q_{n}^{0}$

multiplying the above expressions by $P_{m}(x)$ and $P_{m}^{1}(x)$ respectively, integrating over $0 \leqslant \phi \leqslant \pi$ and using the orthogonal properties of the Legendre Polynomials, we get

$S_{n}^{0}=-\frac{2 n+1}{2} \int_{1}^{-1} P_{n}(x) \bar{w}(x, 0) d x$

$Q_{n}^{0}=-\frac{(2 n+1)(n-1) !}{2(n+1) !} \int_{1}^{-1} P_{n}^{1}(x) \bar{v}(x, 0) d x$

\subsection{Modal shapes}

We can find the modal shape vibrating with upper branch frequency $a_{n}$ by setting to zero the amplitudes corresponding to the lower branch frequency in Eqs. (65) and (66)

$\beta_{n}-b_{n}^{2}=0 ; \quad \alpha_{n}-b_{n}^{2}=0$

therefore

$\beta_{n}=\alpha_{n}$

or

$\frac{\ddot{S}_{n}^{0}}{S_{n}^{0}}=\frac{\ddot{Q}_{n}^{0}}{Q_{n}^{0}}$

Combining the last condition with Eqs. (53) and (54) we get

$p_{n}\left(\frac{S_{n}^{0}}{Q_{n}^{0}}\right)^{2}+\left(q_{n}-r_{n}\right)\left(\frac{S_{n}^{0}}{Q_{n}^{0}}\right)-S_{n}=0$

and solving this quadratic equation
$\Psi_{a_{n}}=\left.\frac{S_{n}^{0}}{Q_{n}^{0}}\right|_{a_{n}}=\frac{r_{n}-q_{n}-\sqrt{\left(q_{n}-r_{n}\right)^{2}+4 p_{n} s_{n}}}{2 p_{n}}$

where the negative sign before the radical is chosen to satisfy either of conditions (73). Finally, the temporal function corresponding to the frequency $a_{n}$ is given by

$S_{n}^{a_{n}}(\tau)=\left.S_{n}^{0}\right|_{a_{n}} \cos \left(a_{n} \tau\right) ; \quad Q_{n}^{a_{n}}(\tau)=\left.Q_{n}^{0}\right|_{a_{n}} \cos \left(a_{n} \tau\right)$

Likewise, we can get the modal shape vibrating with lower frequency $b_{n}$ by setting to zero the amplitudes corresponding to the upper branch frequencies in Eqs. (65) and (66)

$\beta_{n}-a_{n}^{2}=0 ; \quad \alpha_{n}-a_{n}^{2}=0$

leading to

$\Psi_{b_{n}}=\left.\frac{S_{n}^{0}}{Q_{n}^{0}}\right|_{b_{n}}=\frac{r_{n}-q_{n}+\sqrt{\left(q_{n}-r_{n}\right)^{2}+4 p_{n} s_{n}}}{2 p_{n}}$

where the positive sign before the radical is chosen to satisfy either of conditions (79). Therefore, the temporal function corresponding to the frequency $b_{n}$ is given by

$S_{n}^{b_{n}}(\tau)=\left.S_{n}^{0}\right|_{b_{n}} \cos \left(b_{n} \tau\right) ; \quad Q_{n}^{b_{n}}(\tau)=\left.Q_{n}^{0}\right|_{b_{n}} \cos \left(b_{n} \tau\right)$

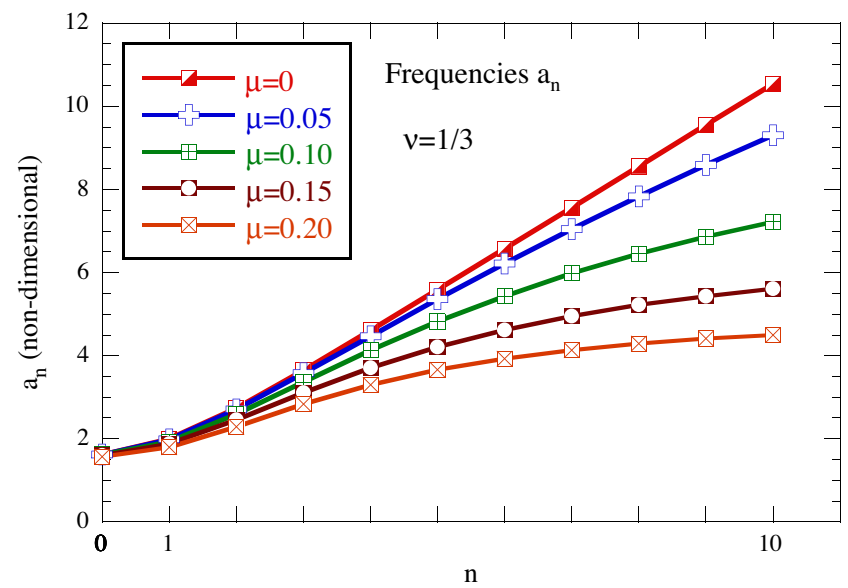

(a)

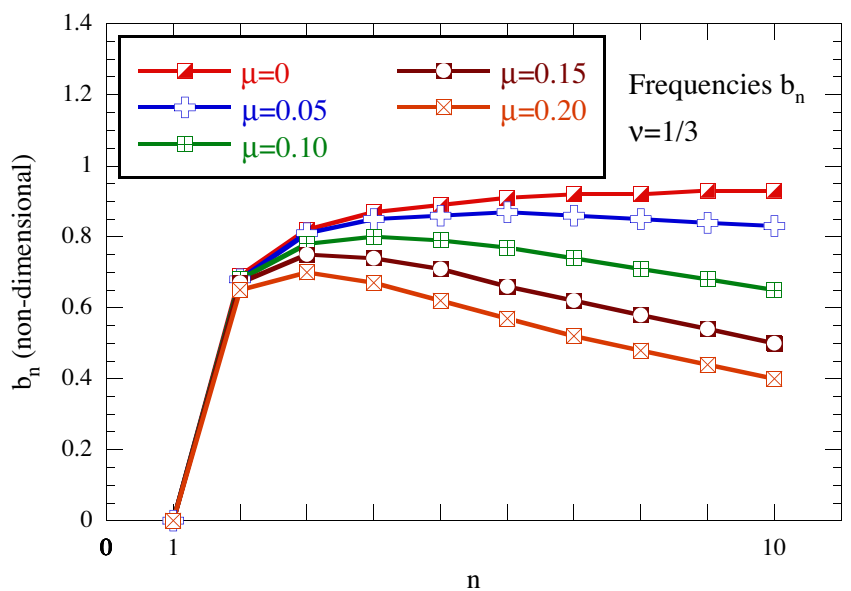

(b)

Fig. 2. Natural frequencies for different values of $\mu$ ( $v=1 / 3$ ). (a) Upper branch $a_{n}$. (b) Lower branch $b_{n}$. 

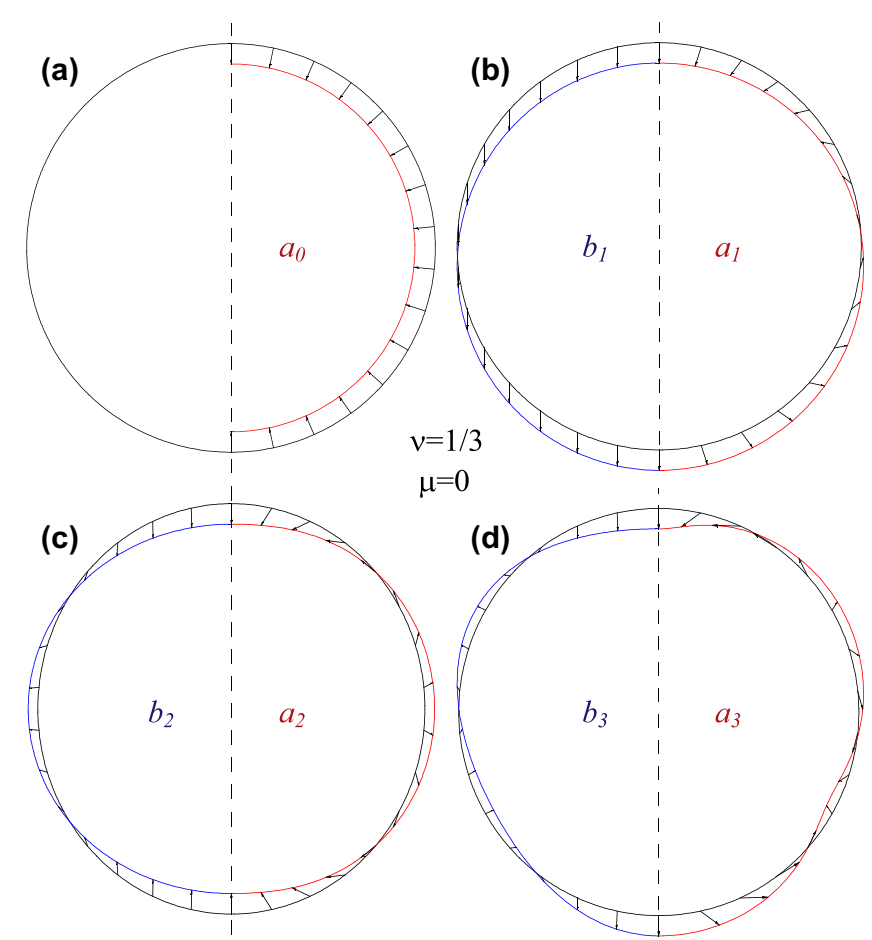

Fig. 3. Modal shapes for: (a) $n=0$, (b) $n=1$, (c) $n=2$, and (d) $n=3 ;(v=1 / 3, \mu=0$, $\epsilon=0.1$ ). Black: undeformed configuration. Red: modal shape for $a_{n}$. Blue: modal shape for $b_{n}$. (For interpretation of the references to color in this figure legend, the reader is referred to the web version of this article.)

The solution of Baker is recovered for $\mu=0$, exceptuating the sign of the expression, which is consistent with the positive direction for the radial displacement taken in the present work.

\section{Analysis of the influence of the nonlocal parameter}

\subsection{Frequencies}

Eqs. (49) and (50) provide the natural frequencies of the free vibration of the shell. It is worth to highlight that the values calculated by Baker [68] are recovered by setting $\mu=0$. As in the local elasticity case, $b_{0}$ leads to a spurious mode, and $b_{1}$ equals zero for every value of the nonlocal parameter thus forecasting a translational mode that will be confirmed afterwards. Fig. 2 shows the frequencies for $n$ from 0 through 10 , for different values of the nonlocal parameter. The frequencies of both upper and lower branches decrease with increasing values of $\mu$. The translational mode $b_{1}$ is accountably independent of the nonlocal parameter, and the breathing mode $a_{0}$ is independent as well. The lower frequencies $a_{1}, a_{2}$, and $b_{2}$ are slightly affected by $\mu$. The effect of the nonlocal parameter starts to play a major role for values of $n \geqslant 3$. Actually there are remarkable differences between local and nonlocal theories for large values of $n$, even for small values of $\mu$.

\subsection{Modal shapes}

To draw the modal shapes, let us fix the initial radial displacement at the north pole to

$w_{n}(0,0)=-\epsilon R$

with $0<\epsilon \ll 1$. Therefore

$\bar{w}_{n}(0,0)=\left.W_{n}\right|_{\phi=0} S_{n}^{0}=\left.W_{n}\right|_{x=1} S_{n}^{0}=-\epsilon$

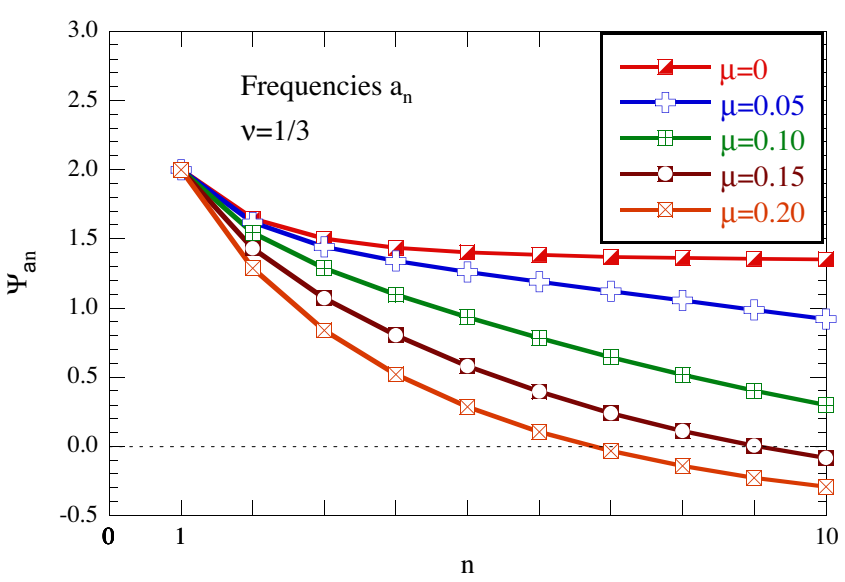

(a)

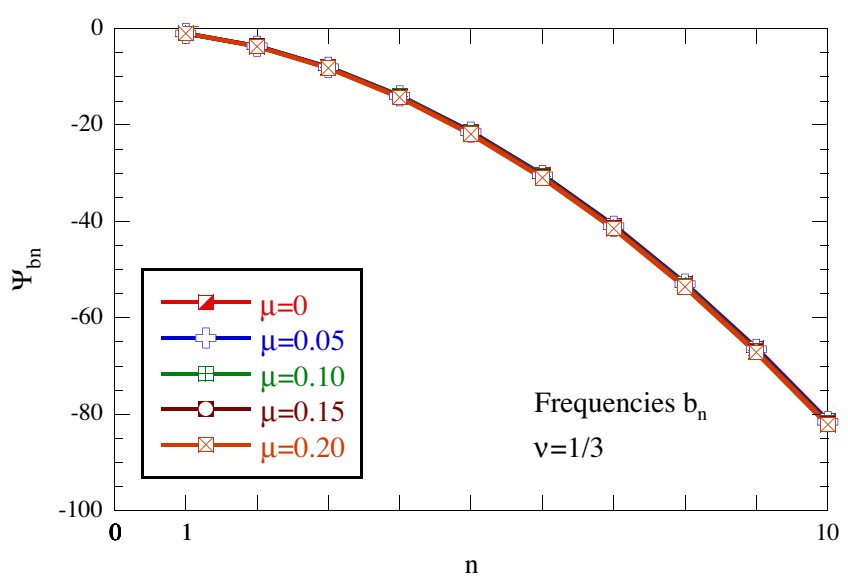

(b)

Fig. 4. Ratio $\phi=0$ for different values of $\mu(v=1 / 3)$. (a) Upper branch $a_{n}$. (b) Lower branch $b_{n}$.

Since $W_{n}=P_{n}$, condition (83) leads to $S_{n}^{0}=-\epsilon$, and the initial amplitudes of the tangential displacement, for frequencies $a_{n}$ and $b_{n}$, are given by

$\left.Q_{n}^{0}\right|_{a_{n}}=-\frac{\epsilon}{\Psi_{a_{n}}} ;\left.\quad Q_{n}^{0}\right|_{b_{n}}=-\frac{\epsilon}{\Psi_{b_{n}}}$

Finally, the modal shapes can be written as

$w_{n}(\phi, 0)=-\epsilon R P_{n}(\cos \phi)$

$v_{n}(\phi, 0)=-\frac{\epsilon}{\Psi_{n}} R P_{n}^{1}(\cos \phi)$

with $\Psi_{n}=\Psi_{a_{n}}$ or $\Psi_{n}=\Psi_{b_{n}}$ depending on the branch of frequencies.

Fig. 3 shows, for $\mu=0$, the modal shapes for $n$ from 0 through 3 . The solution given by Baker [68] is recovered, including the translational mode corresponding to $b_{1}$.

In order to evaluate the influence of the nonlocal parameter on the amplitude of the meridional displacement $v$, the factor $\Psi_{n}$ in Eq. (86) is plotted in Fig. 4 for the upper and lower branches. It can be observed that $\Psi_{a n}$ decreases with $n$ for every value of $\mu$. Moreover, for $\mu>0$, the $\Psi_{a_{n}}$ curves intersect the horizontal axis. Since $v$ increases as the absolute value of $\Psi_{a_{n}}$ decreases, this may lead to large values of the meridional displacement for certain modal shapes, as can be seen in Fig. 5b.

Regarding the lower branch (Fig. 4b), $\Psi_{b_{n}}$ is not affected by the nonlocal parameter. Thus, the nonlocal modal shapes are analogous to their local counterparts. 


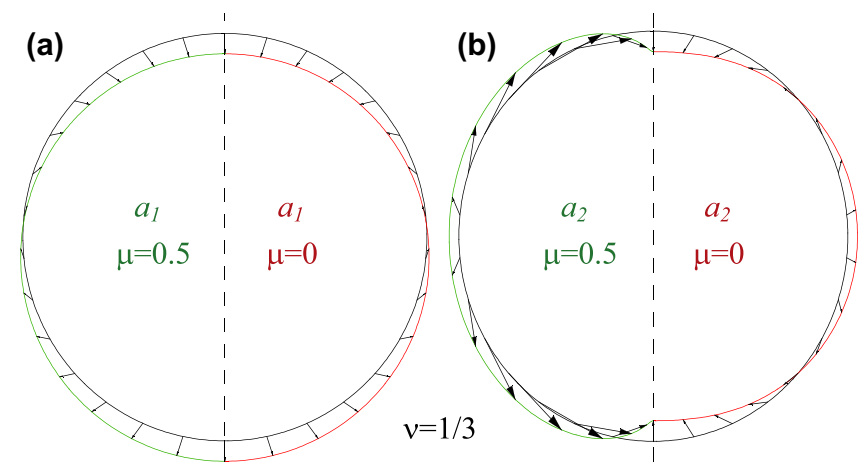

Fig. 5. Modal shapes $a_{n}$ for: (a) $\mathrm{n}=1$ and (b) $\mathrm{n}=2 ;(v=1 / 3, \epsilon=0.1)$. Black: undeformed configuration. Red: modal shape for $\mu=0$. Green: modal shape for $\mu=0.5$. (For interpretation of the references to color in this figure legend, the reader is referred to the web version of this article.)

\section{Conclusions}

A detailed study of free axisymmetric vibrations of a closed spherical nano-shell using the Eringen nonlocal elasticity theory is presented. The hypotheses of thin shells are taken into account and then bending moments, shear efforts and radial normal stresses are neglected.

Using the classical variable separation method, the solution is assumed as a sum of products of both space-dependent and time-dependent functions, permitting to obtain the natural frequencies and modal shapes. As in the local elasticity case, there are two natural frequencies for each value of $n$, the upper branch and the lower branch, with a specific vibration mode for each frequency.

The following conclusions on the effect of nonlocal parameter in natural frequencies and modal shapes can be established:

- The frequencies of both upper and lower branches decrease with increasing values of the nonlocal parameter $\mu$.

- The breathing mode $a_{0}$ is independent of the nonlocal parameter. The lower frequencies $a_{1}, a_{2}$, and $b_{2}$ are slightly affected by $\mu$.

- Remarkable differences between local and nonlocal theories, even for small values of $\mu$, appear for larger values of $n(n \geqslant 3)$.

- The ratio of the radial to meridional displacements, corresponding to certain modal shapes associated to the upper branch, approaches zero for $\mu>0$. This may lead to large values of the meridional displacement in the nonlocal case.

- The modal shapes corresponding to the lower branch frequencies are not affected by the nonlocal parameter, thus the nonlocal modal shapes are analogous to their local counterparts.

\section{Acknowledgements}

The authors are indebted to the Ministerio de Ciencia e Innovación de España (Projects DPI/2011-24068 and DPI/2011-23191) for the financial support.

\section{References}

[1] Kroto H, Heath J, OBrien S, Curl R, Smalley R. C60: buckminsterfullerene Nature 1985;318:162-3.

[2] Iijima S. Helical microtubules of graphitic carbon. Nature 1991;354:56-8.

[3] Martin CR. Membrane-based synthesis of nanomaterials. Chem Mater 1996;8:1739-46.

[4] Drexler KE. Nanosystems: molecular machinery, manufacturing, and computation. New York: Wiley; 1992.
[5] Han J, Globus A, Jaffe R, Deardorff G. Molecular dynamics simulation of carbon nanotubebased gear. Nanotechnology 1997;8:95-102.

[6] Fennimore A, Yuzvinsky TD, Han WQ Fuhrer MS, Cumings J, Zettl A. Rotational actuators based on carbon nanotubes. Nature 2003:408-24.

[7] Bourlon B, Glattli DC, Miko C, Forro L, Bachtold A. Carbon nanotube based bearing for rotational motions. Nano Lett 2004;4:709-12.

[8] Saji V, Choe H, Young K. Nanotechnology in biomedical applications-a review. Int J Nano Biomater 2010;3:119-39.

[9] Wei C, Srivastava D. Nanomechanics of carbon nanofibers: structural and elastic properties. Appl Phys Lett 2004;85:2208-10.

[10] He XQ, Liew KM, Wei C. Mechanical properties of carbon nanocones. Appl Phys Lett 2007;91. 261906-261906-3.

[11] Tsai P, Fang T. A molecular dynamics study of the nucleation, thermal stability and nanomechanics of carbon nanocones. Nanotechnology 2007;18 105702.

[12] Liew KM, Wei JX, He XQ. Carbon nanocones under compression: buckling and post-buckling behaviors. Phys Rev B - Conden Matter Mater Phys 2007;75:195435

[13] Mindlin RD, Tiersten HF. Effects of couple-stresses in linear elasticity. Arch Rat Mech Anal 1962;11(5):415-48.

[14] Kröner E. On the physical reality of torque stresses in continuum mechanics. Int J Eng Sci 1963;1:261-78.

[15] Toupin RA. Elastic materials with couple-stresses. Arch Rat Mech Anal 1963;11(5):385-414.

[16] Toupin RA. Theories of elasticity with couple-stress. Arch Rat Mech Anal 1964;17(2):85-112.

[17] Green AE, Rivlin RS. Multipolar continuum mechanics. Arch Rat Mech Anal 1964;17(2):113-47.

[18] Mindlin RD. Micro-structure in linear elasticity. Arch Rat Mech Anal 1964;16(1):51-78.

[19] Mindlin RD. Second gradient of strain and surface-tension in linear elasticity. Int J Solids Struct 1965;1(4):417-38.

[20] Mindlin RD, Eshel N. On first strain-gradient theories in linear elasticity. Int J Solids Struct 1968;4(1):109-24.

[21] Eringen AC. On differential-equations of nonlocal elasticity and solutions of screw dislocation and surface-waves. J Appl Phys 1983;54(9):4703-10.

[22] Aifantis EC. On the role of gradients in the localization of deformation and fracture. Int J Eng Sci 1992;30(10):1279-99.

[23] Altan SB, Aifantis EC. On the structure of the mode III crack-tip in gradient elasticity. Scripta Metall et Mater 1992;26(2):319-24.

[24] Ru CQ Aifantis EC. A simple approach to solve boundary-value problems in gradient elasticity. Acta Mech 1993;101(1-4):59-68.

[25] Askes H, Gitman I. Review and critique of the stress gradient elasticity theories of Eringen and Aifantis. In: Maugin G, Metrinike A, editors. Mechanics of generalized continua; 2010. p. 203-10.

[26] Askes H, Aifantis EC. Gradient elasticity in statics and dynamics: an overview of formulations, length scale identification procedures, finite element implementations and new results. Int J Solids Struct 2011;48(13): 1962-90.

[27] Eringen AC. Linear theory of nonlocal elasticity and dispersion of plane-waves. Int J Eng Sci 1972;10(5):233-48.

[28] Eringen AC. Nonlocal polar elastic continua. Int J Eng Sci 1972;10:1-16.

[29] Peddieson J, Buchanan GR, McNitt RP. Application of nonlocal continuum models to nanotechnology. Int J Eng Sci 2003;41(3-5):305-12.

[30] Xu MT. Free transverse vibrations of nano-to-micron scale beams. Proc R S A Math Phys Eng Sci 2006;462(2074):2977-95.

[31] Lu P. Dynamic analysis of axially prestressed micro/nanobeam structures based on nonlocal beam theory. J Appl Phys 2007;101(7):073504.

[32] Wang CM, Zhang YY, Ramesh SS, Kitipornchai S. Buckling analysis of microand nano-rods/tubes based on nonlocal timoshenko beam theory. J Phys D Appl Phys 2006;39(17):3904-9.

[33] Wang CM, Zhang YY, He XQ. Vibration of nonlocal timoshenko beams. Nanotechnology 2007;18(10):105401.

[34] Reddy JN. Nonlocal theories for bending, buckling and vibration of beams. Int J Eng Sci 2007;45(2-8):288-307.

[35] Loya J, Lopez-Puente J, Zaera R, Fernandez-Saez J. Free transverse vibrations of cracked nanobeams using a nonlocal elasticity model. J Appl Phys 2009;105(4):044309.

[36] Ke LL, Wang YS, Wang ZD. Nonlinear vibration of the piezoelectric nanobeams based on the nonlocal theory. Compos Struct 2012;94(6):2038-47. http:// dx.doi.org/10.1016/j.compstruct.2012.01.023.

[37] Pradhan SC, Murmu T. Application of nonlocal elasticity and DQM in the flapwise bending vibration of a rotating nanocantilever. Physica E 2010:42:1944-9.

[38] Murmu T, Adhikari S. Scale-dependent vibration analysis of prestressed carbon nanotubes undergoing rotation. J Appl Phys 2010;108:123507.

[39] Narendar S, Gopalakrishnan S. Nonlocal wave propagation in rotating nanotube. Physics 2011;1:17-25.

[40] Aranda-Ruiz J, Loya J, Fernández-Sáez J. Bending vibrations of rotating nonuniform nanocantilevers using the eringen nonlocal elasticity theory. Compos Struct 2012;95:2990-3001.

[41] Sun CT, Zhang HT. Size-dependent elastic moduli of platelike nanomaterials. J Appl Phys 2003;93(2):1212-8.

[42] Murmu T, Pradhan SC. Small-scale effect on the vibration of nonuniform nanocantilever based on nonlocal elasticity theory. Physica E 2009;41(8):1451-6. 
[43] Kiani K. Free longitudinal vibration of tapered nanowires in the context of nonlocal continuum theory via a perturbation technique. Physica E 2010;43(1):387-97.

[44] Narendar S, Gopalakrishnan S. Ultrasonic wave characteristics of nanorods via nonlocal strain gradient models. J Appl Phys 2010;107(8):084312.

[45] Murmu T, Adhikari S. Non local effects in the longitudinal vibration of doublenanorod systems. Physica E 2010;43(1):415-22.

[46] Narendar S. Terahertz wave propagation in uniform nanorods: a nonlocal continuum mechanics formulation including the effect of lateral inertia. Physica E 2011;43(4):1015-20.

[47] Ke L-L, Wang Y-S, Wang Z-D. Non-local elastic plate theories. Proc R Soc A 2008;463:3225-40.

[48] Murmu T, Pradhan SC. Small-scale effect on the free in-plane vibration of nanoplates by nonlocal continuum model. Physica E 2009;41(8):1628-33.

[49] Hosseini-Hashemi S, Zare M, Nazemnezhad R. An exact analytical approach for free vibration of mindlin rectangular nano-plates via nonlocal elasticity. Compos Struct 2013;100:290-9. http://dx.doi.org/10.1016/ j.compstruct.2012.11.035.

[50] Wang Q, Varadan V. Application of nonlocal elastic shell theory in wave propagation analysis of carbon nanotubes. Smart Mater Struct 2007; $16: 178-90$

[51] Wang $\mathrm{Q}$ Wang $\mathrm{C}$. The constitutive relation and small scale parameter of nonlocal continuum mechanics for modelling carbon nanotubes. Nanotechnology 2007;18:075702.

[52] Hua YG, Liew KM, Wang Q He XQ Yakobson BI. Nonlocal shell model for elastic wave propagation in single- and double-walled carbon nanotubes. J Mech Phys Solids 2008;56:3475-85.

[53] Firouz-Abadi R, Fotouhi M, Haddadpour H. Free vibration analysis of nanocones using a nonlocal continuum model. Phys Lett A 2011;375:3593-8.

[54] Wang CM, Duan WH. Vibration analysis of nanorings using nonlocal continuum mechanics and shear deformable ring theory. J Appl Phys 2008;104:014303.

[55] Moosavi H, Mohammadi M, Farajpour A, Shahidi SH. Vibration analysis of nanorings using nonlocal continuum mechanics and shear deformable ring theory. Physica E 2011;44:135-40.

[56] Ghavanloo E, Fazelzadeh S. Radial vibration of free anisotropic nanoparticles based on nonlocal continuum mechanics. Nanotechnology 2013;24:075702.

[57] Fleck NA, Hutchinson JW. Strain gradient plasticity. Advan Appl Mech 1997;33:295-361.

[58] Zhou SJ, Li ZQ. Length scales in the static and dynamic torsion of a circular cylindrical micro-bar. J Shandong Univ Technol 2001;31(5):401-7.

[59] Sudak LJ. Column buckling of multiwalled carbon nanotubes using nonlocal continuum mechanics. J Appl Phys 2003;94(11):7281-7.

[60] Chen Y, Lee JD, Eskandarian A. Atomistic viewpoint of the applicability of microcontinuum theories. Int J Solids Struct 2004;41(8):2085-97.
[61] Heireche H, Tounsi A, Benzair A, Maachou M, Adda Berdia E. Sound wave propagation in single-walled carbon nanotubes using nonlocal elasticity. Physica E 2008;40:2791-9.

[62] Murmu T, Pradhan SC. Thermo-mechanical vibration of a single-walled carbon nanotube embedded in an elastic medium based on nonlocal elasticity theory. Comput Mater Sci 2009;46(4):854-9.

[63] Narendar S, Gopalakrishnan S. Critical buckling temperature of single-walled carbon nanotubes embedded in a one-parameter elastic medium based on nonlocal continuum mechanics. Physica E 2011;43(6):1185-91.

[64] Ansari R, Shahabodini A, Rouhi H. A thickness-independent nonlocal shell model for describing the stability behavior of carbon nanotubes under compression. Compos Struct 2013;100:323-31.

[65] Kessentini S, Barchiesi D. Quantitative comparison of optimized nanorods, nanoshells and hollow nanospheres for photothermal therapy. Biomed Optics Exp 2012;3:590-604.

[66] Fazelzadeh S, Ghavanloo E. Coupled axisymmetric vibration of nonlocal fluidfilled closed spherical membrane shell. Acta Mech 2012;223:2011-20.

[67] Flugge W. Stresses in shells. Berlin: Springer-Verlag; 1960.

[68] Baker W. Axisymmetric modes of vibration of thin spherical shell. The J Acoust Soc Am 1961;33:1749-58.

[69] Brush O, Almroth BO. Buckling of bars, plates and shells. New York: McGrawHill; 1975.

[70] Soedel W. Vibrations of shells and plates. New York: M. Dekker; 1993.

[71] Kahn D, Kim KW. Quantized vibrational modes of nanospheres and nanotubes in the elastic continuum model. J Appl Phys 2001;89:5107-11.

[72] Knoche S, Kierfeld J. Buckling of spherical capsules. Phys Rev E 2011;84:046608.

[73] Tachibana K, Tachibana S. Application of ultrasound energy as a new drug delivery system. Japan J Appl Phys 1999;38:3014.

[74] Wang CQ Wu C. Vibrations of a hollow nanosphere with a porous thin shell in liquid. Macromolecules 2003;36:9285.

[75] Lidmar J, Mirny L, Nelson DR. Virus shapes and buckling transitions in spherical shells. Phys Rev E 2003;68:051910.

[76] Ru C. Buckling of empty spherical viruses under external pressure. J Appl Phys 2009; $105: 124701$.

[77] Ghavanloo E, Fazelzadeh S. Nonlocal elasticity theory for radial vibration of nanoscale spherical shells. Euro J Mech/A Solids 2013. http://dx.doi.org/ 10.1016/j.euromechsol.2013.02.003.

[78] Eringen AC, Edelen DGB. Nonlocal elasticity. Int J Eng Sci 1972;10(3):233-48.

[79] Eringen A. Nonlocal continuum field theories. New York: Springer-Verlag; 2002.

[80] Polyanin A. Handbook of linear partial differential equations for engineers and scientists. Boca Raton: Chapman \& Hall/CRC; 2002.

[81] Arfken G, Weber H, Harris F. Mathematical methods for physicists. Elsevier Academic Press; 2012. 